C. C. YANG AND K. NIINO

KODAI MATH. J.

5 (1982), $84-88$

\title{
ON PRIME ENTIRE FUNCTIONS
}

\author{
By Chung-Chun Yang and KiYoshi Nino
}

1. An entire function $F(z)$ is said to have a factorization with left factor $f(z)$ and right factor $g(z)$ if

$$
F(z)=f(g(z)),
$$

where $f(z)$ and $g(z)$ are entire functions. $F(z)$ is said to be prime (pseudo-prime) if every factorization of form (1) implies that either $f$ or $g$ is linear (polynomial).

Recently many classes of prime entire functions have been exhibited. Moreover, there are several classes of entire functions which and all their derivatives are shown to be prime functions. For instance, it has been proved that $F^{(n)}(z)$ $(n=0,1,2, \cdots)$, where $F(z)=\int_{0}^{z}\left(e^{z}-1\right) e^{z k} d z(k \geqq 2)$, are prime functions. The case, $k=2$ was proved by Urabe-Yang [6] and then Niino [4] proved for $k \geqq 3$. Actually in [4] the following more general result was obtained.

Theorem A. Let $p(z)$ be a polynomial of degree $k(k \geqq 3), H_{1}(z)$ and $H_{2}(z)$ two entire functions which are not identically zero of order less than one. Put

$$
F(z)=\int_{0}^{z}\left\{H_{1}(z) e^{z}+H_{2}(z)\right\} e^{p(z)} d z .
$$

Suppose that $k$ is odd or $p(z)=\alpha_{k} z^{k}\left(\alpha_{k} \neq 0\right)$ when $k$ is even, that all but a finte number of zeros of $H_{1}(z) e^{z}+H_{2}(z)$ are simple and that there are positive number $K$ and integer $N_{0}$ such that for all $l \geqq N_{0}$

$$
\sum_{\jmath \neq l} 1 /\left|a_{\jmath}\left(a_{\jmath}-a_{l}\right)\right| \leqq K
$$

where $\left\{a_{\jmath}\right\}_{j=1}^{\infty}$ are zeros of $H_{1}(z) e^{z}+H_{2}(z)$ other than 0 . Then $F^{(n)}(z)$ is prime for $n=0,1,2, \cdots$.

It was pointed out and exhibited by examples in [4] that the assertion is not always true when $p(z)$ is an arbitrary polynomial of even degree. Also the proof of Theorem A lies heavily on the condition (2) which is quite a cumbersome condition to be checked. In this note, we shall show that the assertion remains valid when condition (2) is removed. Also we shall take this opportunity to clarify some of the argument in [4].

Received September 18, 1980. 
Our main result can be stated as follows.

THEOREM B. Let $p(z)$ be a polynomial of degree $k \geqq 3$ with $p(z+c)-p(-z+c)$ being nonlinear for any constant $c$. Let $H_{1}(z)$ and $H_{2}(z)$ be two entrre functions of order less than 1 and none is identically zero. Put

$$
F(z)=\int_{0}^{z}\left\{H_{1}(z) e^{z}+H_{2}(z)\right\} e^{p(z)} d z
$$

Then $F^{(n)}(z)$ is prime for $n=0,1,2, \cdots$.

2. In order to prove our Theorem, we shall need the following lemmas.

LEMMA 1 (Strelitz [5, p. 432]). Let

$$
\begin{gathered}
R\left(z, \omega, \omega^{\prime}, \cdots, \omega^{(n)}\right)=\sum_{\imath_{0}+\imath_{1}+\cdots+\imath_{n}=0}^{m} R_{\imath_{0} \imath_{1} \cdots \imath_{n}}(z) \omega^{\imath_{0}}\left(\omega^{\prime}\right)^{\imath_{1} \cdots\left(\omega^{(n)}\right)^{\imath_{n}},} \\
P(z, \omega)=\sum_{k=0}^{p} P_{k}(z) \omega^{p-k}, \quad Q(z, \omega)=\sum_{j=0}^{q} Q_{j}(z) \omega^{q-\jmath}
\end{gathered}
$$

where $R_{\imath_{0} \imath_{1} \cdots i_{n}}(z), P_{k}(z)$ and $Q_{j}(z)$ are meromorphic functions. Let $\rho(f)$ denote the order of the meromorphic function $f$ and $\lambda=\max \left\{\rho\left(R_{\imath_{0} \imath_{1} \cdots z_{n}}\right), \rho\left(P_{k}\right), \rho\left(Q_{\jmath}\right)\right\}$ where the maximum is taken over all the possible values of the indices $i_{s}, k$ and $j$. Suppose that $P(z, \omega)$ and $Q(z, \omega)$ are relatively prime in the set of all the polynomials in $\omega$ with meromorphic functions as the coefficients. Furthermore, suppose that $\lambda<\infty$. Then if the differential equation

$$
R\left(z, \omega, \omega^{\prime}, \cdots, \omega^{(n)}\right)=P(z, \omega) / Q(z, \omega)
$$

has a transcendental meromorphic solution $\omega(z)$ of order $\rho>\lambda$, then $Q(z, \omega)$ does not depend on $\omega$.

From the above one can obtain the following:

LEMMA 2. Let $p(z)$ and $\alpha(z)$ be two polynomials with degree of $\alpha(z)>$ degree of $p(z)$. Suppose that $H(z) \equiv H_{1}(z) e^{p(z)}+H_{2}(z)$ with entire functions $H_{i}(z)(\not \equiv 0)$, $\rho\left(H_{\imath}\right)<\rho\left(e^{p(z)}\right)(i=1,2)$. If $F(z)=\int_{0}^{z} H(z) e^{\alpha(z)} d z \equiv Q(g(z))$ for some polynomial $Q$ and entire function $g$, then $Q$ has to be linear.

Proof. Suppose that

$$
F(z)=Q(g(z)),
$$

where $Q(z)$ is a polynomial and $g(z)$ is transcendental entire function. Differentiating (3) twice we obtain successively

$$
F^{\prime}(z) \equiv H(z) e^{\alpha(z)} \equiv Q^{\prime}(g) g^{\prime}
$$

and 


$$
F^{\prime \prime}(z) \equiv\left[H^{\prime}(z)+H(z) \alpha^{\prime}(z)\right] e^{\alpha(z)} \equiv Q^{\prime}(g) g^{\prime \prime}+Q^{\prime \prime}(g) g^{\prime 2} .
$$

It follow, by taking the quotient of (4) and (5) that

or

$$
\frac{Q^{\prime}(g) g^{\prime \prime}+Q^{\prime \prime}(g) g^{\prime 2}}{Q^{\prime}(g) g^{\prime}}=\frac{H^{\prime}(z)+H(z) \alpha^{\prime}(z)}{H(z)}
$$

$$
g^{\prime}=\frac{Q^{\prime}(g)}{Q^{\prime \prime}(g)}\left\{\frac{H^{\prime}(z)+H(z) \alpha^{\prime}(z)}{H(z)}-\frac{g^{\prime \prime}(z)}{g^{\prime}(z)}\right\} \equiv \frac{P(z, g)}{Q(z, g)} .
$$

Now from (4) and the assumption that $\rho(H)<\rho\left(e^{\alpha}\right)$, one concludes easily that $\rho\left(g^{\prime \prime} / g^{\prime}\right)<\rho(g)$. Therefore, Strelitz's lemma is applicable and $Q(z, g)$ does not depend on $g$ if $P(z, g)$ and $Q(z, g)$ are relative prime in $g$. Hence $Q^{\prime \prime}(g)$ must be a constant unless $Q^{\prime \prime}(z)=c_{2}(z-d)^{m}$ and $Q^{\prime}(z)=c_{1}(z-d)^{m+1}$ for some constants $c_{1}, c_{2}$ and $d$, and nonnegative integer $m$. But then we have $g^{\prime} \equiv a(z) g+b(z)$ for some meromorphic functions $a(z)$ and $b(z)$ satisfying $T(r, a(z))=o(T(r, g))=$ $T(r, b(z))$ as $r \rightarrow \infty$. Now if $Q^{\prime}(z)$ is not a constant, then from (4) one must have that $-b(z) / a(z) \equiv d$ (according to the extension of Nevanlinna's second fundamental Theorem for deficient functions [3, p. 47]). Thus we have

Hence

$$
c_{1}(g-d)^{m+1} a(z)(g-d) \equiv H(z) e^{\alpha(z)}
$$

$$
c_{1}(g-d)^{m+2} \equiv \frac{H(z)}{a(z)} e^{\alpha(z)}
$$

or

$$
c_{1}(g-d)^{m+2} e^{-\alpha(z)}+\left(\frac{-H_{1}(z)}{a(z)}\right) e^{p(z)} \equiv \frac{H_{2}(z)}{a(z)}
$$

or

$$
c_{1}\left[(g-d) e^{-\alpha(z) /(m+2)}\right]^{m+2}+\left(\frac{-H_{1}(z)}{a(z)}\right) e^{p(z)} \equiv \frac{H_{2}(z)}{a(z)}
$$

It follows that the function $-H_{1}(z) e^{p(z)} / a(z)$ attains the deficient function $-H_{2}(z) / a(z)$ at points with multiplicities greater than or equal to $m+2 \geqq 2$. This is impossible [3, p. 47]. Hence $m+1$ has to be zero and therefore $Q^{\prime}(z)$ is a constant. Thus $Q(z)$ is linear as asserted.

3. Proof of Theorem $B$. Clearly the function $F(z)$ satisfies $\delta\left(0, F^{\prime}\right)=1$. Hence it is pseudo-prime [2]. Therefore we shall only need to consider $F(z)=$ $p(g(z))$ or $F(z)=g(p(z))$ for some polynomial $p(z)$ and transcendental entire function $g(z)$. The former case can be ruled out by Lemma 2. Therefore, we shall concentrate on the later case. This is the case where the argument used in [4] which needs some clarification. The proof will be exactly the same as in [4] except we want to clarify the assertion about the statement $\rho_{N(r, 0, H ; \Delta)}<1$. ( $\left[4\right.$, p. 182]). Actually we need to show that if $H_{1}(z)$ and $H_{2}(z)$ are of order than one, then 


$$
\rho_{N(r, 0, H ; \Delta)} \leqq \max \left\{\rho_{N\left(r, 0, H_{1}\right)}, \rho_{N\left(r, 0, H_{2}\right)}\right\}<1,
$$

In order to prove the above statement we recall the following fact:

LEMMA $3[1$, p. 52]. If $f(z)$ is an entire function of growth $(\rho, \sigma)$, then for every positive $\varepsilon$ and $\eta$ and $k>1$, for sufficiently large $R$, we have

$$
\log |f(z)|>-B(\sigma+\varepsilon)(k R)^{\rho}, \quad|z|<R .
$$

except in a set of circles the sum of whose radii at most $2 \eta k R ; B$ depends only on $\eta$ and $k$.

Now put $\Delta=\Delta_{\delta}^{(1)} \cup \Delta_{\delta}^{(2)}, \Delta_{\delta}^{(1)}=\{z ; 0 \leqq \arg z<\pi / 2-\delta\} \cup\{z ; 3 \pi / 2+\delta<\arg z<2 \pi\}$, $\Delta_{\delta}^{(2)}=\{z ; \pi / 2+\delta<\arg z<3 \pi / 2-\delta\}$. If $z \in \Delta_{\delta^{*}}^{(2)}\left(0<\delta^{*}<\delta\right)$, then there is a constant $\tau\left(0<\tau<\sin \delta^{*}\right)$ such that

$$
\left|H_{1}(z) e^{z}\right| \leqq e^{-\tau r}
$$

holds for large $r=|z|$. We apply Lemma 3 to $H_{2}(z)$. We take $\eta$ and $k(>1)$ satisfying $0<6 \eta k<\delta-\delta^{*}$. Then there are constants $\rho(0<\rho<1)$ and $A$ such that

$$
\log \left|H_{2}(z)\right| \geqq-A r^{\rho}
$$

holds for large $r=|z|$, except in a set of circles enclosing of zeros of $H_{2}(z)$ the sum of whose radii at most $2 \eta k r$. Let $\left\{D_{n}^{*}\right\}$ be the family of domains consisting of the above circles enclosing zeros of $H_{2}(z)$ in $\Delta_{\delta^{*}}^{(2)}$ and $\left\{D_{n}\right\}$ the subfamily of $\left\{D_{n}^{*}\right\}$ satisfying $D_{n} \cap \Delta_{\delta}^{(2)} \neq \phi$. Then it follows that $D_{n} \subset \Delta_{\delta^{*}}^{(2)}$. Hence we have, for $z \in\left\{\overline{\bar{U}_{\delta}^{(2)}-\cup D_{n}}\right\} \cup\left\{\cup \partial D_{n}\right\}$

$$
\left|H_{2}(z)\right| \geqq \exp \left(-A r^{\rho}\right)>\exp (-\tau r) \geqq\left|H_{1}(z) e^{z}\right|,
$$

and consequently $H(z)=H_{2}(z)+H_{1}(z) e^{z} \neq 0$ for large $|z|=r, z \in \overline{\Delta_{\delta}^{(2)}-\cup D_{n}}$. It follows from (7) and Rouché's theorem that $H(z)$ and $H_{2}(z)$ has the same number of zeros inside $D_{n}$. Hence $n\left(r, 0, H ; \Delta_{\delta}^{(2)}\right) \leqq n\left(r, 0, H_{2} ; \Delta_{\delta^{*}}^{(2)}\right)+O(1) \leqq n\left(r, 0, H_{2}\right)+$ $O(1)$ for large $r$.

Similarly we have $n\left(r, 0, H ; \Delta_{\delta}^{(1)}\right) \leqq n\left(r, 0, H_{1} ; \Delta_{\delta^{*}}^{(1)}\right)+O(1)$. Hence $(6)$ is proved.

The rest part will be exactly the same as in [4] and this also completes the proof of Theorem B.

\section{REFERENCES}

[1] BoAs, R.P., Entire Functions, Academic Press, 1954.

[2] Goldstein, R., On factorization of certain entire functions, J. London Math. Soc. 2 (1970), 221-224.

[3] Hayman, W.K., Meromorphic Functions, Oxford Clarendon Press, 1964.

[4] Nino, K., On prime entire functions, J. Math. Anal. Appl. 66 (1978), 178-187.

[5] Strelitz, Sh., On meromorphic solutions of algebraic differential equations, Trans. Amer. Math. Soc. 258 (1980), 431-440. 
[6] URABE, H. AND C.-C. YANG, On certain entire functions which together with their derivative are prıme, Kōdai Math. Sem. Rep. 29 (1977), 167-178.

Chung-Chun Yang

Naval Research Laboratory

DEPARTMENT OF THE NAVY

WASHINGTON, D.C. 20375

U.S.A.
KiYOSHI NinO

Faculty of Technology

KaNAZAWA UnIVERSITY

Kodatsuno, Kanazawa 920

JAPAN 